ARTIGO ESPECIAL

SPecial Article

\title{
Espondiloartropatias: Outras Artropatias ${ }^{(*)}$
}

\author{
Sampaio-Barros PD, Carvalho MAP, Azevedo VF, Campos WR, Carneiro SCS, Giorgi RDN, Gonçalves \\ CR, Hilário MOE, Keiserman MW, Leite NH, Pereira IA, Vieira WP, Vilela EG, Xavier RM, Ximenes AC
}

\section{DESCRIÇÃO DO MÉTODO DE COLETA DE EVIDÊNCIAS}

Reunião consensual para elaboração do texto com inclusão das citações bibliográficas, numa colaboração de reumatologistas com as especialidades de reumatologia pediátrica, dermatologia, gastroenterologia e oftalmologia. Foram convidados 12 reumatologistas responsáveis pelo ambulatório de espondiloartropatias em suas instituições (ou seus representantes); cada participante foi convidado a fazer uma análise crítica, utilizando o conceito da medicina baseada em evidências, de um aspecto diferente dentro do espectro do tratamento das espondiloartropatias. Foi convidado um especialista de cada área para fazer a mesma análise crítica nos tópicos espondiloartropatias juvenis (reumatologista pediátrico), psoríase (dermatologista), doenças inflamatórias intestinais (gastroenterologista) e uveíte anterior (oftalmologista). Após a elaboração de um texto-base de apoio para as discussões, todos os especialistas se reuniram, no período entre 22 e 24 de abril de 2004, a fim de discutir individualmente cada um dos 16 tópicos elaborados e estabelecer um consenso baseado em evidências, a partir do qual os coordenadores redigiram o texto do consenso, submetido à apreciação de todos os participantes para a realização dos ajustes finais.

\section{GRAU DE RECOMENDAÇÃO E FORÇA DE EVIDÊNCIA}

A: Estudos experimentais e observacionais de melhor consistência.

B: Estudos experimentais e observacionais de menor consistência.

C: Relatos de casos, estudos não controlados.

D: Opinião desprovida de avaliação crítica, baseada em consensos, estudos fisiológicos ou modelos animais.

\section{OBjetivos}

Oferecer informações sobre a otimização do tratamento das diferentes espondiloartropatias.

\section{CONFLito DE INTERESSE}

Os autores Sampaio-Barros PD, Carneiro SCS, Hilário MOE, Xavier RM e Ximenes AC declararam vínculo com a Indústria Farmacêutica.

\section{INTRODUÇÃO}

O conceito das espondiloartropatias soronegativas foi estabelecido em 1974, quando os pesquisadores ingleses Moll e Wright propuseram que se englobasse dentro de um mesmo conjunto algumas doenças até então consideradas completamente distintas entre si, mas que na verdade apresentavam diversas características comuns. Estas características englobavam aspectos clínicos (dor axial inflamatória, associada à artrite, predominante em grandes articulações de membros inferiores, e entesopatias peri- féricas), radiológicos (sacroiliíte) e laboratoriais (soronegatividade para o fator reumatóide, pois, até a década de 70, alguns pesquisadores consideravam a espondilite anquilosante como o componente axial da artrite reumatóide), em indivíduos com predisposição genética (ligada ao antígeno de histocompatibilidade HLA-B27). Este conjunto incluiu a espondilite anquilosante, a artrite psoriásica, a artrite reativa e a síndrome de Reiter, e as artropatias enteropáticas (associadas às doenças inflamatórias intestinais ${ }^{(1)}(\mathbf{C})$. Nos últimos 20 anos, devido ao crescente número de pacientes que apresentam um diagnóstico provável,

\footnotetext{
* Trabalho realizado por representantes da Sociedade Brasileira de Reumatologia. Elaboração final: 21 de junho de 2004.

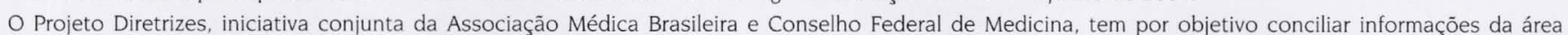

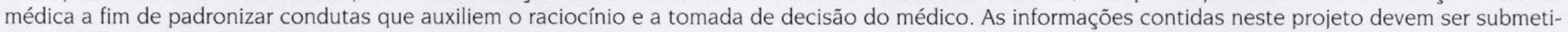

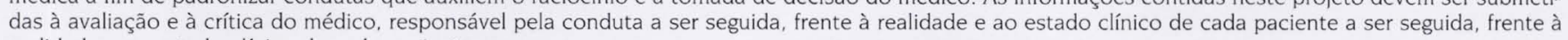
realidade e ao estado clínico de cada paciente.
} 
mas que não conseguem preencher os critérios diagnósticos para uma doença definida dentro do grupo, foi criado o conceito das espondiloartropatias indiferenciadas. Recentemente, especialistas internacionais propuseram a mudança de nome para espondiloartropatias, suprimindo o termo soronegativas $^{(2)}(\mathbf{C})$.

\section{CONSIDERAÇÕES GERAIS SOBRE A ABORDAGEM TERAPÊUTICA DE PACIENTES COM ESPONDILOARTROPATIAS.}

As doenças crônicas, como as espondiloartropatias, na maioria das vezes causam um sentimento de desamparo e inutilidade mais assustador do que a doença em si. Por isso, o paciente sempre deve ser informado de forma realista sobre as perspectivas prognósticas de sua doença, perspectivas essas que são freqüentemente otimistas, desde que haja uma abordagem global de atendimento. O paciente deve estar absolutamente ciente de que sua participação - especialmente no estar bem consigo mesmo, apesar da doença - será um ponto de partida fundamental para o sucesso terapêutico. O seguimento dos pacientes e o tratamento devem ser realizados de forma individual, caso a caso, já que a história natural da doença pode passar por flutuações durante seu curso evolutivo. $\mathrm{O}$ médico deve considerar o impacto da doença para o indivíduo dentro de sua comunidade, com as devidas implicações no seu ambiente familiar e de trabalho, além dos aspectos sociais e financeiros. Apesar de inexistir, até o momento, um tratamento específico para a cura, deve-se ressaltar que os recursos terapêuticos disponíveis possibilitam, de um modo geral, um adequado controle da doença. Um ponto fundamental para tais conquistas está centrado numa boa relação médico-paciente; quando necessário, o médico assistente deve indicar auxílio psicoterápico ou uso de drogas antidepressivas.

\section{SÍNDROME DE REITER / ARTRITE REATIVA}

O termo Síndrome de Reiter, proposto em 1942 por Bauer e Engleman, atualmente está restrito aos casos caracterizados pela tríade uretrite, artrite e conjuntivite, que ocorre após infecção gênito-urinária ou gastrointestinal; está incluído no grupo das artrites reativas. O moderno conceito de artrite reativa dentro do grupo das espondiloartropatias requer a presença de uma oligoartrite associada à evidência de infecção precedente ${ }^{(3)}(\mathbf{D})$.

$\mathrm{O}$ agente infeccioso representa, no indivíduo geneticamente predisposto, o "fator gatilho" para o desenvol- vimento da doença. Na etiologia pós-disentérica, predominam as bactérias enteropatogênicas, como Shigella (flexneri, sonnei), Salmonella (typhimurium, enteritidis), Yersinia (enterocolitica, pseudotuberculosis) e Campylobacter (jejuni); já nos casos de etiologia gênito-urinária, predomina a Chlamydia trachomatis ${ }^{(4)}(\mathbf{D})$. Numerosos fatores imunológicos concorrerão para o desencadeamento da doença. Com relação à interação agente infecciosohospedeiro, ocorre invasão das células mucosas, com replicação intracelular do agente infeccioso; existe uma eliminação antigênica deficiente, permitindo que antígenos bacterianos se liguem a macrófagos e monócitos (com função de células apresentadoras de antígenos) e atinjam a circulação, ativando as imunidades humoral e celular. Em muitos casos, é observada uma diminuição dos níveis de citocinas Th1, indispensáveis para a eliminação de inúmeros microrganismos. Em alguns casos de doença crônica, podem ser detectados determinantes antigênicos (geralmente lipopolissacárides de membrana, de Chlamydia, Shigella ou Salmonella) na biópsia de membrana sinovial; a persistência destes determinantes antigênicos, mesmo na ausência do microrganismo vivo, poderia ser responsável pela perpetuação da doenç ${ }^{(5)}($ D) .

Os sintomas clínicos costumam se iniciar de uma a quatro semanas após infecção (na etiologia entérica é comum quadro diarréico autolimitado, por vezes disentérico, enquanto que, na etiologia gênito-urinária, ocorre uretrite com sintomatologia leve). Dentro da tríade característica da doença, a primeira manifestação costuma ser a uretrite, freqüentemente serosa, com descarga matinal, oligosintomática; seu diagnóstico é difícil nas mulheres, e pode evoluir com prostatite (muitas vezes assintomática) nos homens acometidos. A conjuntivite costuma ser leve, de resolução espontânea, durando sete a dez dias, sem deixar seqüelas. O quadro articular costuma ser caracterizado por uma oligoartrite assimétrica, recorrente, de predomínio em grandes articulações de membros inferiores, sendo comum a presença de volumosos derrames articulares recorrentes em joelhos, em pacientes com doença ativa. Na evolução do quadro articular, é bastante freqüente a presença de entesites na inserção do tendão de Aquiles e da fáscia plantar, também cursando com tenossinovites em dedos de pés e mãos (os chamados "dedos em salsicha"). Acometimento axial (espondilite) pode aparecer em cerca de $20 \%$ dos casos. Comprometimento visceral é pouco freqüente. Pode apresentar evolução como surto único (10\% a 20\%), crises recorrentes $(40 \%$ a $60 \%)$ ou cronificar $(20 \%$ a $30 \%)$. A pesquisa do HLA-B27, positiva em $50 \%$ a $80 \%$ dos casos, é impor- 
tante não como diagnóstico, mas como determinante de prognóstico da doença ${ }^{(6)}(\mathbf{D})$.

Na infância, a maioria dos casos de artrite reativa ocorre em meninos de 8 a 12 anos, estando associada predominantemente às infecções entéricas. Já nos adolescentes, a artrite reativa posterior à infecção genital por Chlamydia ocorre com maior freqüência.

Tratamento. $\mathrm{Na}$ fase articular aguda da doença, iniciase o tratamento com AINH, em doses convencionais; o tratamento deverá durar enquanto houver atividade articular da doença, não havendo nenhum AINH comprovadamente superior aos demais ${ }^{(7)}($ D) . Os corticosteróides costumam ser utilizados em casos de oligoartrite ou entesopatia persistentes não responsivas aos AINH (prednisona, na dose de 5 a $10 \mathrm{mg} /$ dia, ou equivalente) ou monoartrite persistente (triancinolona hexacetonide intraarticular) ${ }^{(7)}(\mathbf{D})$.

Nos casos que evoluem para doença articular crônica, predominantemente periférica, podem ser utilizados a sulfasalazina ${ }^{(8)}(\mathbf{A})$ e o metotrexato ${ }^{(9)}(\mathbf{C})$, nas doses convencionais. Em pacientes com artrite reativa onde se considera iniciar o uso de droga imunossupressora deve-se solicitar exame de HIV, pois alguns pacientes aidéticos podem apresentar quadro clínico de Síndrome de Reiter como manifestação inicial e, se tratados com imunossupressores, podem desencadear a doença em toda sua expressão.

$\mathrm{O}$ uso dos agentes biológicos infliximabe e etanercepte ainda não está bem definido nas artrites reativas.

A administração de antibióticos deve ser sempre considerada quando a infecção está presente, sendo a sua eficácia dependente do agente causal da moléstia. Nas infecções entéricas causadas por Salmonella, Shigella e Campylobacter, só há indicação de tratamento quando a infecção não é autolimitada; nestes casos, pode-se usar a ciprofloxacina $(500$ $\mathrm{mg}$, via oral, duas vezes por dia) ou sulfametoxazol-trimetoprin, durante sete a dez dias ${ }^{(10)}(\mathbf{D})$. Em pacientes com infecção ativa do trato gênito-urinário por Chlamydia, devese administrar tratamento antibiótico com tetraciclina $(500 \mathrm{mg}$, via oral, três vezes por dia) ou derivados (doxiciclina ou minociclina) durante 14 dias $^{(10)}(\mathrm{D})$; é imprescindível o tratamento dos parceiros para se evitar reinfestações. Como a infecção urogenital por Chlamydia é muitas vezes assintomática, ou cursa com sintomas leves (geralmente não referidos pelos pacientes), e sendo a Chlamydia um importante fator causal de prostatite crônica (assintomática em 50\% dos casos) e salpingite crônica (causa não hormonal freqüente de infertilidade feminina), é prudente que se investigue pacientes com diagnóstico de artrite reativa sem fator causal definido. Não existem evidências de que o uso crônico de antibióticos seja eficaz nas artrites reativas, nem o uso da ciprofloxacina $^{(11)}(\mathbf{A})$, nem da doxiciclina ${ }^{(12)}(\mathbf{A})$.

\section{ARTRITES ENTEROPÁTICAS}

As doenças inflamatórias intestinais podem acometer de 2 a 20 indivíduos em cada 200.000 pessoas; já o acometimento articular pode afetar $2 \%$ a $26 \%$ dos pacientes com a doença. Classicamente, podemos observar manifestações articulares associadas à retocolite ulcerativa e à doença de Crohn, e, em menor proporção, à doença de Whipple, doença celíaca e cirurgia de bypass intestinal ${ }^{(13)}(\mathbf{D})$.

Os acometimentos articulares podem ser subdivididos em oligoartrite periférica, poliartrite periférica e espondilite enteropática. A oligoartrite periférica acomete preferencialmente grandes articulações de membros inferiores, associada a entesopatias periféricas (notadamente em inserção de tendão aquileano e fáscia plantar); não tem predomínio de sexo ou antígeno de histocompatibilidade específico, e sua evolução está invariavelmente associada à atividade da doença intestinal; lesões cutâneas, tipo eritema nodoso, podem ocorrer em $10 \%$ a $25 \%$ destes pacientes. Uma poliartrite periférica, geralmente não deformante, pode ocorrer na doença de Whipple e após cirurgia de bypass intestinal; quadro semelhante, porém potencialmente mais agressivo e deformante, pode ocorrer em pacientes com doença de Crohn; também não apresentam associação com HLA específico, e sua evolução costuma ser independente do acometimento intestinal. A espondilite enteropática pode acometer $2 \%$ a $12 \%$ dos pacientes com retocolite ulcerativa e doença de Crohn; predomina no sexo masculino (2-3:1), sendo que $50 \%$ a $75 \%$ destes pacientes apresentam HLA-B27 positivo; o quadro clínico e radiológico da espondilite enteropática é semelhante ao observado na espondilite anquilosante, e sua evolução costuma ser independente do quadro intestinal ${ }^{(14)}(\mathrm{D})$.

Tratamento. Devido à possibilidade de causarem ulceração ou sangramento intestinais, confundindo o médico sobre a atividade intestinal da doença, os antiinflamatórios não hormonais não são prescritos de rotina em pacientes com artrite enteropática ${ }^{(13)}(\mathrm{D})$. É preferível o uso de corticosteróides em baixas doses (prednisona $5-10 \mathrm{mg} / \mathrm{dia}$, ou equivalente) para o alívio da artrite ou entesopatia periféricas; o uso de doses elevadas (até $1 \mathrm{mg} / \mathrm{kg} /$ dia de prednisona, ou equivalente) somente é indicado nos casos de inflamação intestinal importante ${ }^{(15)}(\mathrm{D})$. As drogas de base mais utilizadas para o tratamento dos surtos de atividade são os derivados da 5-ASA, a mesalazina e a sulfasalazina; é 
importante salientar que a sulfasalazina e o derivado asacol ou asalit da mesalazina têm ação somente sobre o intestino grosso, enquanto que o derivado pentasa tem ação sobre os intestinos delgado e grosso ${ }^{(16)}(\mathbf{C})$. Nos casos mais graves de recorrência da doença de Crohn ou doença intestinal extensa ou formação de múltiplas fístulas, o uso do metotrexato $^{(17)}(\mathbf{A})$, da azatioprina ${ }^{(18)}(\mathbf{B})$ e do infliximabe ${ }^{(19)}(\mathbf{A})$ pode ser indicado.

\section{ESPONDILOARTROPATIAS INDIFERENCIADAS}

As espondiloartropatias indiferenciadas englobam um grupo de pacientes que apresentam características clínicas e/ou radiológicas sugestivas de uma espondiloartropatia, mas que não preenchem os critérios diagnósticos de nenhuma das doenças definidas dentro do grupo. Muitas vezes representa um diagnóstico provisório, englobando um conjunto bastante heterogêneo de pacientes, que pode variar de um estágio precoce ou uma forma abortiva ou frustrada de uma espondiloartropatia definida ${ }^{(20)}(\mathbf{D})$. Estudos controlados de longo prazo indicaram que um paciente com dor inflamatória em sacroilíacas pode demorar até 14 anos para desenvolver uma sacroiliíte radiológica, permitindo então ser feito o diagnóstico de espondilite ${ }^{(21)}(\mathbf{C})$. A presença do HLA-B27 representa um fator prognóstico de evolução para uma doença definida ${ }^{(22)}(\mathbf{C})$. Nos pacientes que preenchem os critérios de classificação para espondiloartropatias do Grupo Europeu de Estudo das Espondiloartropatias ${ }^{(23)}(\mathbf{B})$ e não preenchem os critérios diagnósticos para uma doença definida, esta pode ser considerada indiferenciada.

Tratamento. $O$ tratamento é o mesmo das doenças definidas dentro do grupo, enfatizandose que as formas indiferenciadas costumam ter curso evolutivo mais leve e com menos complicações que as outras doenças do grupo. $\mathrm{O}$ uso do infliximabe ${ }^{(24)}(\mathbf{C})$ e do etanercepte ${ }^{(25)}(\mathbf{C})$ apresenta resultados promissores.

\section{UVEÍTE ANTERIOR}

As uveítes anteriores, não granulomatosas, com crises agudas geralmente unilaterais e recorrentes, são encontradas com freqüência dentro do grupo das espondiloartropatias. Podem ocorrer em até $40 \%$ dos pacientes com espondilite, sendo menos freqüientes nas outras doenças do grupo ${ }^{(26)}(\mathbf{D})$. Podem representar o primeiro sintoma clínico de uma espondiloartropatia indiferenciada ${ }^{(27)}(\mathbf{C})$. Nas formas crônicas, recidivantes, podem ser encontradas alterações no segmento posterior, tais como vitreíte, edema de mácula e edema do disco óptico ${ }^{(28)}($ D). A presença do HLA-B27 geralmente não indica pior prognóstico oftalmológico, e sim maior possibilidade de desenvolver sintomas articulares ${ }^{(29)}(\mathbf{B})$ e evoluir para espondilite ${ }^{(30)}(\mathbf{C})$; as uveítes anteriores associadas ao HLA-B27 são mais freqüentes em homens e tendem a aparecer em indivíduos mais jovens ${ }^{(31)}(\mathbf{C})$.

Tratamento. Quando um paciente com espondiloartropatia apresenta uma crise aguda de uveíte anterior, deve-se encaminhá-lo a um oftalmologista para confirmar o diagnóstico. Na confirmação da crise de uveíte anterior, o oftalmologista indicará o uso de corticosteróide tópico (para diminuir a inflamação) e midriáticos (para dilatar a pupila); o uso de corticoterapia sistêmica deve ser reservado aos casos graves e naqueles com recorrência das crises $^{(26)}($ D $)$.

O uso da sulfasalazina parece diminuir o número e a intensidade das crises de uveíte anterior nos pacientes com espondilite ${ }^{(32)}(\mathbf{A})$, embora esta afirmação seja controversa entre os oftalmologistas. Nas formas recidivantes, o uso de corticosteróides por via oral está indicado para o controle da uveíte $^{(28)}(\mathbf{D})$. Nos raros casos de uveíte crônica, o uso de drogas imunossupressoras como a azatioprina, o metotrexato e a ciclosporina, em doses convencionais, isoladas ou associadas, pode ser indicado em algumas situações (necessidade de dose de manutenção de corticosteróide elevada por tempo prolongado, contra-indicação ao uso do corticosteróide, inflamação crônica refratária, acometimento de pólo posterior) ${ }^{(28)}(\mathrm{D})$. O uso dos antibióticos por longo prazo nas uveítes anteriores não mostrou resultados satisfatórios ${ }^{(33)}(\mathbf{A})$. Estudos iniciais (abertos e com pequena casuística) analisando o uso dos agentes biológicos etanercepte ${ }^{(34)}(\mathbf{C})$ e infliximabe ${ }^{(35)}(\mathbf{C})$ no tratamento das uveítes têm demonstrado resultados interessantes no controle da inflamação a curto prazo; estudos controles a longo prazo são necessários para se estabelecer sua real eficácia.

\section{ESPONDILOARTROPATIAS JUVENIS}

As espondiloartropatias juvenis (que se iniciam em indivíduos menores de 16 anos) costumam ter características clínicas que permitem diferenciá-las da doença que se inicia no adulto. Primordialmente, apresentam franco predomínio de acometimento de articulações periféricas, geralmente como uma oligoartrite de articulações de membros inferiores, associada à entesopatia periférica; o acometimento axial costuma ser tardio, muitas vezes já na fase adulta ${ }^{(36)}(\mathbf{D})$. É comum o diagnóstico de espondiloartropatia indiferen- 
ciada, como a Síndrome SEA, caracterizada por uma artrite com entesopatia periférica, soronegativa para o FAN e fator reumatóide, que, em número significativo de casos, costuma evoluir para espodilite ${ }^{(37)}(\mathbf{B})$.

Tratamento. O uso de AINH é recomendado no início do tratamento, em doses habituais, enquanto houver atividade de doença; não existe um AINH específico que seja comprovadamente mais eficaz que os outros ${ }^{(36)}(\mathbf{D})$. O uso de corticosteróides somente costuma ser indicado nos casos potencialmente incapacitantes e não responsivos ao $\mathrm{AINH}$, podendo ser utilizado por via oral ou intra-articular (nos casos de monoartrite persistente) ${ }^{(38)}(\mathbf{B})$.

\section{REFERÊNCIAS}

1. Moll JM, Haslock I, Macrae IF, Wright V. Associations between ankylosing spondylitis, psoriatic arthritis, Reiter's disease, the intestinal arthropathies, and Behcet's syndrome. Medicine 53:3 43-64, 1974

2. Braun J, Sieper J. Building consensus on nomenclature and disease classification for ankylosing spondylitis: results and discussion of a questionnaire prepared for the International Workshop on New Treatment Strategies in Ankylosing Spondylitis, Berlin, Germany, 18-19 January 2002. Ann Rheum Dis 61(Suppl 3):61-7, 2002.

3. Kingsley G, Sieper J. Third International Workshop on Reactive Arthritis, 23-26 September 1995, Berlin, Germany. Ann Rheum Dis 55: $564-84,1996$.

4. Toivanen P, Toivanen A. Two forms of reactive arthritis? Ann Rheum Dis 58:737-41, 1999.

5. Granfors K. Do bacterial antigens cause reactive arthritis? Rheum Dis Clin North Am 18:37-48, 1992;.

6. Amor B. Reiter's syndrome: diagnosis and clinical features. Rheum Dis Clin North Am 24:677-95, 1998.

7. Palazzi C, Olivieri I, D’Amico E, Pennese E, Petricca A. Management of reactive arthritis. Expert Opin Pharmacother 51:61-70, 2004

8. Clegg DO, Reda DJ, Weisman MH, et al. Comparison of sulfasalazine and placebo in the treatment of reactive arthritis (Reiter's syndrome). A Department of Veterans Affairs Cooperative Study. Arthritis Rheum 39:2021-7, 1996

9. Lally EV, Ho G Jr. A review of methotrexate therapy in Reiter syndrome. Semin Arthritis Rheum 15: 139-45, 1985.

10. Sieper J, Braun J. Treatment of reactive arthritis with antibiotics. $\mathrm{Br}$ J Rheumatol 37:717-20, 1998.

11. Sieper J, Fendler C, Laitko S, et al. No benefit of long-term ciprofloxacin treatment in patients with reactive arthritis and undifferentiated oligoarthritis: a three-month, multicenter, doubleblind, randomized, placebo controlled study. Arthritis Rheum 42:1386-96, 1999.

12. Smieja M, MacPherson DW, Kean W, et al. Randomised, blinded, placebocontrolled trial of doxycycline for chronic seronegative arthritis. Ann Rheum Dis 60:1088-94, 2001.

13. Holden W, Orchard T, Wordsworth P. Enteropathic arthritis. Rheum Dis Clin North Am 29:513-30, 2003.
A sulfasalazina, na dose de 30 a $50 \mathrm{mg} / \mathrm{kg} / \mathrm{dia}$, apresenta bons resultados no componente periférico da doença ${ }^{(39)}(\mathbf{B})$. O metotrexato e a ciclosporina (isolados ou associados) apresentam bons resultados na artrite reumatóide juvenil, mas ainda carecem de estudos controle nas espondiloartropatias juvenis ${ }^{(38)}(\mathrm{D})$.

Quanto aos agentes biológicos, infliximabe e etanercepte, estudos abertos e com casuísticas pequenas mostram resultados favoráveis em uma proporção significativa dos pacientes $^{(40)}($ D) . É importante lembrar que as vacinas de vírus vivos são contra-indicadas em pacientes em tratamento com imunossupressores ou agentes biológicos.

14. Smale S, Natt RS, Orchard TR, Russell AS, Bjarnason I. Inflammatory bowel disease and spondylarthropathy. Arthritis Rheum 44:2728-36, 2001.

15. Podolsky DK. Inflammatory bowel disease. N Engl J Med 347: 417-29, 2002.

16. Hanauer SB, Krawitt EL, Robinson M, Rick GG, Safdi MA. Longterm management of Crohn's disease with mesalamine capsules (Pentasa). Pentasa Crohn's Disease Compassionate Use Study Goup. Am J Gastroenterol 88:1343-51, 1993

17. Feagan BG, Rochon J, Fedorak RN, et al. Methotrexate for the treatment of Crohn's disease. N Engl J Med 332:292-7, 1995.

18. Bouhnik Y, Lemman M, Mary JY, et al. Long-term follow-up of patients with Crohn's disease treated with azathioprine or 6mercaptopurine. Lancet 347:215-9, 1996.

19. Hanauer SB, Feagan BG, Lichtenstein GR, et al. Maintenance infliximab for Crohn's disease: the ACCENT 1 randomised trial. Lancet 359:1541-9, 2002.

20. Zeidler H, Mau W, Khan MA. Undifferentiated spondyloarthropathies. Rheum Dis Clin North Am 18:187-202, 1992.

21. Mau W, Zeidler H, Mau R, et al. Clinical features and prognosis of patients with possible ankylosing spondylitis: results of a 10-year follow-up. J Rheumatol 15:1109-14, 1988.

22. Sampaio-Barros PD, Conde RA, Donadi EA, et al. Undifferentiated spondyloarthropathies in Brazilians: importance of HLA-B27 and the B7-CREG alleles in characterization and disease progression. J Rheumatol 30:2632-7, 2003.

23. Dougados M, van der Linden S, Juhlin R, H, et al. The European Spondylarthropathy Study Group preliminary criteria for the classification of spondylarthropathy. Arthritis Rheum 34:1218-27, 1991.

24. Brandt J, Haibel H, Reddig J, Sieper J, Braun J. Successful short term treatment of severe undifferentiated spondyloarthropathy with the anti-tumor necrosis factor-alpha monoclonal antibody infliximab. J Rheumatol 29:118-22, 2002.

25. Brandt J, Khariouzov A, Listing J, et al. Successful short term treatment of patients with severe undifferentiated spondyloarthritis with the anti-tumor necrosis factoralpha fusion receptor protein etanercept. J Rheumatol 31:531-8, 2004.

26. Bañares A, Hernandez-Garcia C, Fernandez-Gutierrez B, Jover JA. Eye involvement in the spondyloarthropathies. Rheum Dis Clin North Am 24:771-84, 1998. 
27. Pato E, Bañares A, Jover JA, et al. Undiagnosed spondyloarthropathy in patients presenting with anterior uveitis. J Rheumatol 27: 2198-202, 2000 .

28. Oréfice F, Carvalho MAP, Moreira C. Espondiloartropatias. In: Oréfice F, editor. Uveíte clínica e cirúrgica, Texto e Atlas. 2a ed. Rio de Janeiro: Editora Cultura Médica; 2004.

29. Linssen A, Meenken C. Outcomes of HLA-B27-positive and HLAB27 negative acute anterior uveitis. Am J Ophthalmol 120: 351-61, 1995.

30. Carvalho MAP, Campos WR, Araújo CAA, Lacerda RR, Oréfice F. Uveítes anteriores não granulomatosas, espondiloartropatias e HLA-B27. Rev Bras Reumatol 39:195-202, 1999.

31. Monnet D, Breban M, Hudry C, Dougados M, Brezin AP. Ophthalmic findings and frequency of extraocular manifestations in patients with HLA-B27 uveitis: a study of 175 cases. Ophthalmology 111:802-9, 2004.

32. Benitez-Del-Castilho JM, Garcia-Sanchez J, Iradier T, Bañares A. Sulfasalazine in the prevention of anterior uveitis associated with ankylosing spondylitis. Eye 14:340-3, 2000.

33. Wakefield D, McCluskey P, Verma M, Aziz K, Gatus B, Carr G. Ciprofloxacin treatment does not influence course or relapse rate of reactive arthritis and anterior uveitis. Arthritis Rheum 42: 1894-7, 1999;.
34. Smith JR, Levinson RD, Holland GN, et al. Differential efficacy of TNF inhibition in the management of inflammatory eye disease and associated rheumatic disease. Arthritis Rheum 45:252-7, 2001.

35. El-Shabrawi Y, Hermann J. Anti-tumor necrosis factor alpha therapy with infliximab as an alternative to corticosteroids in the treatment of human leukocyte antigen B27-associated acute anterior uveitis. Ophthalmology 109:2342-6, 2002.

36. Burgos-Vargas R, Pacheco-Tena C, Vazquez-Mellado J. Juvenileonset spondyloarthropathies. Rheum Dis Clin North Am 23: 569-98, 1997.

37. Cabral DA, Oen KG, Petty RE. SEA syndrome revisited: a longterm followup of children with a syndrome of seronegative enthesopathy and arthropathy. J Rheumatol 19:1282-5, 1992.

38. Huang F, Zhang J, Zhu J, Guo J, Yang C. Juvenile spondyloarthropathies: the Chinese experience. Rheum Dis Clin North Am 29:531-47, 2003.

39. Burgos-Vargas R, Vazquez-Mellado J, Pacheco-Tena C, HernandezGarduno A, Goycochea-Robles MV. A 26 week, randomised, double-blind, placebo controlled exploratory study of sulfasalazine in juvenile-onset spondyloarthropathies. Ann Rheum Dis 61: 941-2, 2002.

40. Reiff AHM. Prolonged efficacy of etanercept in refractory juvenile ankylosing spondylitis. Arthritis Rheum 44:S292, 2001. 